\title{
T cell protein tyrosine phosphatase (TCPTP) deficiency in muscle does not alter insulin signalling and glucose homeostasis in mice
}

\author{
K. Loh • T. L. Merry • S. Galic • B. J. Wu • M. J. Watt • \\ S. Zhang $\cdot$ Z.-Y. Zhang $\cdot$ B. G. Neel $\cdot$ T. Tiganis
}

Received: 22 September 2011 / Accepted: 25 October 2011 /Published online: 29 November 2011

(C) Springer-Verlag 2011

\begin{abstract}
Aims/hypothesis Insulin activates insulin receptor protein tyrosine kinase and downstream phosphatidylinositol-3kinase (PI3K)/Akt signalling in muscle to promote glucose uptake. The insulin receptor can serve as a substrate for the protein tyrosine phosphatase (PTP) 1B and $\mathrm{T}$ cell protein
\end{abstract}

K. Loh $\cdot$ T. L. Merry $\cdot$ S. Galic $\cdot$ B. J. Wu $\cdot$ T. Tiganis $(\bowtie)$

Department of Biochemistry and Molecular Biology,

Monash University,

Melbourne, VIC 3800, Australia

e-mail: Tony.Tiganis@med.monash.edu.au

M. J. Watt

Department of Physiology, Monash University,

Melbourne, VIC, Australia

S. Zhang $\cdot$ Z.-Y. Zhang

Department of Biochemistry and Molecular Biology,

Indiana University School of Medicine,

Indianapolis, IN, USA

B. G. Neel

Campbell Family Cancer Research Institute,

Ontario Cancer Institute, Princess Margaret Hospital,

Toronto, ON, Canada

B. G. Neel

Department of Medical Biophysics, University of Toronto,

Toronto, ON, Canada

Present Address:

S. Galic

St Vincent's Institute,

Melbourne, VIC, Australia

Present Address:

B. J. Wu

Heart Research Institute,

Camperdown, NSW, Australia tyrosine phosphatase (TCPTP), which share a striking $74 \%$ sequence identity in their catalytic domains. PTP1B is a validated therapeutic target for the alleviation of insulin resistance in type 2 diabetes. PTP1B dephosphorylates the insulin receptor in liver and muscle to regulate glucose homeostasis, whereas TCPTP regulates insulin receptor signalling and gluconeogenesis in the liver. In this study we assessed for the first time the role of TCPTP in the regulation of insulin receptor signalling in muscle.

Methods We generated muscle-specific TCPTP-deficient (Mck-Cre;Ptpn $\left.2^{l o x / l o x}\right)$ mice (Mck, also known as Ckm) and assessed the impact on glucose homeostasis and muscle insulin receptor signalling in chow-fed versus high-fat-fed mice.

Results Blood glucose and insulin levels, insulin and glucose tolerance, and insulin-induced muscle insulin receptor activation and downstream PI3K/Akt signalling remained unaltered in chow-fed Mck-Cre;Ptpn $2^{\text {lox } / \text { ox }}$ versus Ptpn $2^{\text {lox/lox }}$ mice. In addition, body weight, adiposity, energy expenditure, insulin sensitivity and glucose homeostasis were not altered in high-fat-fed Mck-Cre;Ptpn $2^{\text {lox/lox }}$ versus Ptpn $2^{\text {lox/lox }}$ mice.

Conclusions/interpretation These results indicate that TCPTP deficiency in muscle has no effect on insulin signalling and glucose homeostasis, and does not prevent high-fat diet-induced insulin resistance. Thus, despite their high degree of sequence identity, PTP1B and TCPTP contribute differentially to insulin receptor regulation in muscle. Our results are consistent with the notion that these two highly related PTPs make distinct contributions to insulin receptor regulation in different tissues.

Keywords Diabetes · Glucose homeostasis · Insulin resistance $\cdot$ Insulin signalling $\cdot$ Muscle $\cdot$ Protein tyrosine phosphatase $\cdot$ PTP $\cdot$ PTP1B $\cdot$ TCPTP 


$\begin{array}{ll}\text { Abbreviations } \\ \text { GTT } & \text { Glucose tolerance test } \\ \text { HFF } & \text { High-fat fed } \\ \text { ITT } & \text { Insulin tolerance test } \\ \text { JAK } & \text { Janus kinase } \\ \text { MET } & \text { Met proto-oncogene tyrosine kinase } \\ \text { PI3K } & \text { Phosphatidylinositol-3-kinase } \\ \text { PTK } & \text { Protein tyrosine kinase } \\ \text { PTP } & \text { Protein tyrosine phosphatase } \\ \text { STAT } & \text { Signal transducer and activator of transcription } \\ \text { TCPTP } & \text { T cell protein tyrosine phosphatase }\end{array}$

\section{Introduction}

Type 2 or non-insulin-dependent diabetes accounts for 90 $95 \%$ of cases of diabetes and is characterised by insulin resistance and hyperglycaemia [1, 2]. Insulin resistance manifests as diminished insulin responsiveness in insulin's key target tissues, including liver, fat and muscle, and is associated with ageing, obesity and a sedentary lifestyle $[1,2]$. Insulin is secreted by the pancreas in response to an increase in blood glucose levels and suppresses glucose production in the liver, while promoting glucose uptake into muscle and adipose tissue. Insulin exerts its effects by binding to the insulin receptor on the surface of cells. Binding of insulin activates the protein tyrosine kinase (PTK) activity of the intracellular $\beta$-subunit, resulting in autophosphorylation and the phosphorylation of substrates such as IRS-1 [1, 3, 4]. Phosphotyrosyl residues on IRS-1 serve as docking sites for the recruitment of Src homology (SH)2 domain-containing proteins; these include the adaptor protein growth factor receptor-bound protein 2 (GRB-2) and the p85 regulatory subunit of phosphatidylinositol-3-kinase (PI3K), which activate the Ras/mitogen-activated protein kinase and PI3K/ Akt cascades to elicit the metabolic and mitogenic actions of insulin $[1,3,4]$. In particular, PI3K signalling and Akt2 are essential for the translocation/docking of the GLUT4 glucose transporter on the plasma membrane in muscle and fat $[1,2,5-7]$. Although the precise molecular mechanism(s) underlying the development of insulin resistance remain unclear, one widely accepted mechanism involves the impairment of PI3K recruitment to IRS-1 [1, 2]. Hence, strategies that enhance insulin receptor activation and downstream signalling may be effective in alleviating insulin resistance.

Protein tyrosine phosphatases (PTPs) [8] are key regulators of insulin receptor signalling, dephosphorylating and inactivating the insulin receptor within minutes of stimulation [9]. The prototypic family member PTP1B (encoded by PTPN1) dephosphorylates the insulin receptor $\beta$ subunit Y1162/Y1163 activation loop autophosphorylation site to attenuate insulin signalling in vivo [10-15]. Mice with a global deficiency in PTP1B $\left(P t p n 1^{-/}\right)$exhibit enhanced insulin sensitivity, associated with increased insulin receptor activation and downstream IRS-1 tyrosine phosphorylation in liver and muscle [11-13, 15]. PTP1B's direct contribution to insulin receptor regulation in muscle has been substantiated by the generation of muscle-specific PTP1B-deficient (Mck-Cre;Ptpn $\left.1^{l o x} / o x\right)$ mice (Mck, also known as $\left.C k m\right)$ [15]. These mice exhibit improved insulin sensitivity and glucose tolerance, associated with elevated muscle insulin receptor activation and signalling [15]. Moreover, Mck-Cre;Ptpn $1^{\text {lox/lox }}$ mice are protected from the development of insulin resistance induced by a high-fat diet [15]. Conversely, transgenic overexpression of $P T P 1 B$ in muscle causes insulin resistance [16], and PTP1B protein levels and/or activity may be increased in muscle and adipose tissue of insulin-resistant humans and rodents [17-20]. PTP1B's prominent role in insulin receptor regulation and glucose homeostasis has engendered considerable interest from the pharmaceutical industry in the development of PTP1B inhibitors for the treatment of type 2 diabetes [19, 21, 22].

Several other tyrosine-specific PTPs have also been implicated in the attenuation of insulin signalling in muscle. Like PTP1B, leucocyte common antigen-related (LAR) phosphatase levels may be increased in muscle from insulin-resistant rodents/humans, and transgenic overexpression of LAR phosphatase in muscle suppresses insulin signalling and glucose uptake, and promotes whole-body insulin resistance [17, 23-27]. Recent studies have also shown that insulin receptor activation and signalling are elevated in PTP $\varepsilon$-deficient differentiated myoblasts, but the role of PTPE in glucose homeostasis in vivo remains unknown [28].

Previously, we have shown that the insulin receptor can also serve as a bona fide substrate for the $\mathrm{T}$ cell protein tyrosine phosphatase (TCPTP; encoded by PTPN2) [8, 10, $29,30]$. In particular, we reported that insulin receptor activation and signalling were enhanced in TCPTPdeficient fibroblasts and in HepG2 hepatoma cells after TCPTP knockdown [29, 30]. We also reported that TCPTP knockdown in PTP1B-deficient mouse embryonic fibroblasts further enhanced insulin receptor activation, consistent with the notion that the two PTPs act together to attenuate insulin signalling [10]. PTP1B and TCPTP are also similarly oxidised by reactive oxygen species in fibroblasts in response to insulin [30]. The concerted oxidation of PTP1B and TCPTP suggests that both enzymes attenuate insulin receptor signalling in a coordinated manner [31]. Finally, we showed that TCPTP may also regulate insulin signalling and glucose homeostasis in vivo [32]. We reported that insulin signalling was elevated in hepatocytes isolated from TCPTP heterozygous mice $\left(P t p n 2^{+/-}\right)$and that high-fat fed (HFF) Ptpn $2^{+/-}$mice were 
protected from developing hyperglycaemia [32]. The prevention of hyperglycaemia in HFF $P t p n 2^{+/}$mice could be attributed to the decreased hepatic gluconeogenesis and glucose production associated with enhanced signal transducer and activator of transcription (STAT) 3 phosphorylation, as well as to prolonged hepatic insulin-induced PI3K/ Akt signalling [32]. Previous studies have shown that PTP1B deficiency in liver also enhances insulin receptor activation and the insulin-induced suppression of hepatic glucose production [33]. Thus, the combinatorial inhibition of PTP1B and TCPTP in liver might be an effective strategy for preventing fasting hyperglycaemia in patients with type 2 diabetes.

Skeletal muscle accounts for the majority of glucose disposal following a meal, and insulin resistance in muscle is an early event in the pathogenesis of type 2 diabetes. Therefore, the inhibition of PTPs such as PTP1B, which attenuate insulin receptor activation and signalling in muscle, might be particularly effective in alleviating insulin resistance. Given the established potential for PTP1B and TCPTP to act together in attenuating insulin receptor signalling in fibroblasts and hepatocytes [29, 30, 32], we asked whether TCPTP might also regulate the insulin response and glucose homeostasis in muscle.

\section{Methods}

Antibodies and reagents Human insulin was from SigmaAldrich (St Louis, MO, USA) and the rat insulin RIA kit from Linco Research (St Charles, MO, USA). Rabbit $\alpha$-phospho-Y1162/1163 insulin receptor was from Biosource International (Camarillo, CA, USA). $\alpha$-Phospho-S473-Akt, $\alpha$-phospho-Y705-STAT3, and $\alpha$-Akt and mouse $\alpha$-STAT3 were from Cell Signaling (Beverly, MA, USA). Mouse $\alpha$-insulin receptor $\beta$ subunit was from Santa Cruz (Santa Cruz, CA, USA), $\alpha$-tubulin (Ab-5) from Sigma-Aldrich and $\alpha$-TCPTP (6F3) from Medimabs (Montréal, QC, Canada).

Mice We maintained mice on a $12 \mathrm{~h}$ light-dark cycle in a temperature-controlled high barrier facility (ARL, Monash University, Melbourne, VIC, Australia) with free access to food and water according to NHMRC Australian Code of Practice for the Care and Use of Animals. Ptpn $2^{\text {lox/lox }}$ $(\mathrm{C} 57 \mathrm{BL} / 6)$ mice were crossed with mice expressing Cre recombinase under control of the muscle creatine kinase promoter (Mck-Cre; C57Bl/6) to excise Ptpn2 in muscle. Mck-Cre mice have been described previously [15] and were backcrossed on to the $\mathrm{C} 57 \mathrm{BL} / 6$ background for six generations. A detailed description of the construct design and generation of Ptpn $2^{l o x / 4}$ mice has been provided elsewhere [34]. Briefly, the targeting construct incorporated loxP sites flanking exons 5 and 6 , and a neomycin-resistance cassette flanked by FLP recombinase target (FRT) sites. The linearised construct was electroporated into Bruce 4 (C57BL/6J) embryonic stem cells and G418-resistant colonies identified by Southern blot analysis. Correctly targeted clones were injected into BALB/c blastocytes. Highpercentage male chimeras were mated with $\mathrm{C} 57 \mathrm{BL} / 6 \mathrm{~J}$ mice to produce Ptpn $2^{l o x / 4}$ offspring and then bred with FLP mice (C57BL/6) to excise the neomycin-resistance cassette. We used aged- and sex-matched mice for all experiments. Mice were fed standard chow (19\% protein by weight, $4.6 \%$ fat and $4.8 \%$ crude fibre $)$ or a high-fat diet $(23.5 \%$ fat by weight; $46 \%$ of total energy from fat; SF04-027; Specialty Feeds, Glen Forest, WA, Australia) as indicated.

Metabolic measurements Insulin tolerance tests (ITTs) and glucose tolerance tests (GTTs) were performed as described previously [35]. Fed and fasted blood was collected by retro-orbital bleeding and blood glucose plasma insulin levels determined as described previously [35]. To measure oxygen consumption and energy expenditure, food intake and ambulatory activity mice were acclimatised for $24 \mathrm{~h}$ and then monitored for $48 \mathrm{~h}$ in an environmentally controlled system (Comprehensive Lab Animal Monitoring System; Columbus Instruments, Columbus, OH, USA) fitted with indirect open circuit calorimetry, and food consumption and activity monitors. Respiratory exchange ratios $\left(\dot{V} \mathrm{CO}_{2} / \dot{V} \mathrm{O}_{2}\right)$ were calculated from the gas exchange data. Data were averaged for two dark and two light cycles.

2-Deoxy-D-glucose uptake assay Soleus muscles dissected from 8-week-old mice were incubated for $20 \mathrm{~min}$ in oxygenated $\left(95 \% \quad \mathrm{O}_{2} / 5 \% \quad \mathrm{CO}_{2}\right)$ Krebs-Henseleit buffer supplemented with $0.1 \%$ (wt/vol.) BSA, $8 \mathrm{mmol} / 1$ mannitol, $2 \mathrm{mmol} / \mathrm{l}$ pyruvate plus $10 \mu \mathrm{mol} / \mathrm{l} 2$-deoxy-D-glucose, and 2-deoxy-D-[1,2- $\left.{ }^{3} \mathrm{H}\right]$ glucose $\left(1.85 \times 10^{4} \mathrm{~Bq} / \mathrm{ml}\right)$ (GE Healthcare, Mount Prospect, IL, USA) uptake was determined for $10 \mathrm{~min}$ in the presence or absence of $10 \mathrm{nmol} / \mathrm{l}$ insulin (Actrapid; Novo Nordisk, Bagsværd, Denmark) as described previously [36].

Cell culture Primary mouse myoblasts were isolated from the muscle of 2- to 4-week-old mice and cultured in Ham's F10 medium containing 20\% (vol./vol.) heat-inactivated FBS, antibiotics (100 U/ml penicillin, $100 \mu \mathrm{g} / \mathrm{ml}$ streptomycin) and $2.5 \mathrm{ng} / \mathrm{ml}$ human basic fibroblast growth factor as described previously [35]. Mouse myoblasts were differentiated for 5 days into multinucleated myotubes in DMEM containing 5\% (vol./vol.) horse serum (Sigma-Aldrich) plus antibiotics, and then serum-starved for $6 \mathrm{~h}$ and stimulated with insulin. Rat L6 myoblasts (American Type Culture Collection, Manassas, VA, USA) were cultured in DMEM containing $20 \%$ (vol./vol.) heat-inactivated FBS plus antibiotics. Where indicated, L6 myoblasts were serum-starved for $4 \mathrm{~h}$, 
pre-incubated with PTP1B inhibitor (compound II [37]) and/or TCPTP inhibitor (compound 8; [38]) for $1 \mathrm{~h}$, and then stimulated with $1 \mathrm{nmol} / 1$ insulin.

Biochemical analyses Mouse tissues were dissected and immediately frozen in liquid $\mathrm{N}_{2}$. Tissues were homogenised in 10 to 20 volumes of ice-cold RIPA lysis buffer and clarified by centrifugation as described previously [35]. Cells were lysed in RIPA buffer and clarified by centrifugation $\left(16,000 \mathrm{~g}\right.$ for $5 \mathrm{~min}$ at $\left.4^{\circ} \mathrm{C}\right)$. Tissue and cell lysates were resolved by SDS-PAGE, transferred on to Immobilon-P transfer membrane (Millipore, Billerica, MA, USA) and immunoblotted as described previously [39]. Lox/lox and muscle-specific TCPTP-deficient tissue and cell lysates, or cells treated with vehicle control or inhibitor were resolved on the same SDS-PAGE gel or otherwise transferred on to the same transfer membrane for immunoblotting and development.

Statistical analyses All data are presented as mean \pm SEM, and statistical significance was determined using the Student's $t$ test or repeated-measures two-way ANOVA. A value of $p<0.05$ was considered significant.

\section{Results}

Generation and characterisation of mice with musclespecific deletion of TCPTP To investigate whether TCPTP plays a role in the regulation of insulin receptor signalling in muscle, we generated a floxed allele of Ptpn2 (loxP sites flanking exons 5 and 6) by gene targeting in Bruce 4 (C57BL/6J) embryonic stem cells. Ptpn2 $2^{\text {lox/lox }}(\mathrm{C} 57 \mathrm{BL} / 6)$ mice were crossed with mice expressing the Cre recombinase under control of the muscle creatine kinase promoter (Mck-Cre; C57Bl/6) to excise Ptpn2 in muscle. TCPTP production, as determined by immunoblot analysis, was deleted in the gastrocnemius and soleus muscles of the Mck-Cre;Ptpn $2^{\text {loxlox }}$ mice, but not in other insulin-responsive tissues such as liver, fat and hypothalamus (Fig. 1a).

At 8 weeks of age, no differences in body weight were noted between Mck-Cre;Ptpn $2^{\text {lox/lox }}$ and Ptpn $2^{\text {lox/lox }}$ male mice (Fig. 1b). Consistent with this, corresponding tissue weights, including gastrocnemius muscle, were similar in both groups (Fig. 1c). No overt differences in muscle development or glycogen content (as assessed by haematoxylin and eosin, and periodic acid-Schiff's reagent staining) were evident in Mck-Cre;Ptpn $2^{\text {lox } / l o x}$ mice (Fig. 1d). Oxygen consumption, energy expenditure (as assessed by indirect calorimetry) and respiratory exchange ratios (a measure of fat and carbohydrate utilisation) were unaltered between Mck-Cre;Ptpn $2^{\text {lox/lox }}$ and Ptpn $2^{\text {lox/lox }}$ male mice, suggesting that TCPTP deficiency in muscle had no impact on whole-body energy homeostasis and fuel use (Fig. 1e-g). Moreover, ambulatory activity was not affected in mice lacking TCPTP in muscle when compared with Ptpn $2^{l o x / l o x}$ littermates (Fig. 1h).

Insulin sensitivity and glucose homeostasis are not altered in muscle-specific TCPTP-deficient mice To investigate whether TCPTP deficiency in muscle improves insulin sensitivity and whole-body glucose clearance, we performed ITTs and GTTs in 8-week-old Mck-Cre;Ptpn $2^{\text {lox/lox }}$ versus Ptpn $2^{l o x / o x}$ mice. No differences in ITT (Fig. 2a, b) or GTT (Fig. 2c, d) results were evident in Mck-Cre; Ptpn $2^{\text {lox/lox }}$ versus Ptpn $2^{\text {lox/lox }}$ mice. Consistent with these results, we found no significant differences in fed and fasted blood glucose levels, or fed and fasted plasma insulin levels in Mck-Cre;Ptpn $2^{\text {lox/lox }}$ versus Ptpn $2^{\text {lox/lox }}$ mice (Fig. 2e, f). In addition, no differences were evident in the uptake of 2deoxy-D-glucose into Mck-Cre; Ptpn $2^{\text {lox/lox }}$ versus Ptpn $2^{\text {lox/lox }}$ soleus muscle ex vivo in response to insulin (Fig. 2g). These results indicate that muscle-specific TCPTP deficiency in chow-fed lean mice does not alter insulin sensitivity and glucose homeostasis.

Insulin signalling is not altered by TCPTP deficiency in muscle Next we determined whether TCPTP deficiency alters insulin signalling in muscle (Fig. 3). First, we determined whether insulin receptor activation and signalling were altered by TCPTP deficiency in gastrocnemius muscle after bolus insulin administration $(1 \mathrm{mU} / \mathrm{g}, 10 \mathrm{~min}$; intraperitoneal). Insulin receptor activation and downstream PI3K/Akt signalling were assessed by immunoblot analysis using antibodies to the insulin receptor $\beta$-subunit Y1162/ Y1163 and Akt Ser-473 phosphorylation sites respectively. No differences in basal or insulin-induced insulin receptor activation and PI3K/Akt signalling were evident in muscle in vivo (Fig. 3a). Second, we isolated myoblasts from Mck-Cre;Ptpn $2^{\text {lox/lox }}$ and Ptpn $2^{\text {lox/lox }}$ mice, and assessed insulin-induced signalling in the corresponding differentiated myotubes (Fig. 3b); the differentiation of myoblasts into myotubes, as assessed by the abundance of myogenic determination factor (MYOD) and myogenin, was unaltered by TCPTP deficiency (data not shown). We found that insulin-induced signalling (1-10 nmol/1; 0-30 min), as assessed by Akt Ser-473 phosphorylation, was not altered by TCPTP deficiency in myotubes in vitro (Fig. 3b; data not shown). Taken together, these results indicate that TCPTP deficiency does not alter insulin signalling in muscle.

Body weight and glucose homeostasis are not altered in highfat fed muscle-specific TCPTP-deficient mice Although insulin sensitivity was unaltered in chow fed Mck-Cre; $P t p n 2^{l o x / l o x}$ mice, it is possible that differences might only 
a

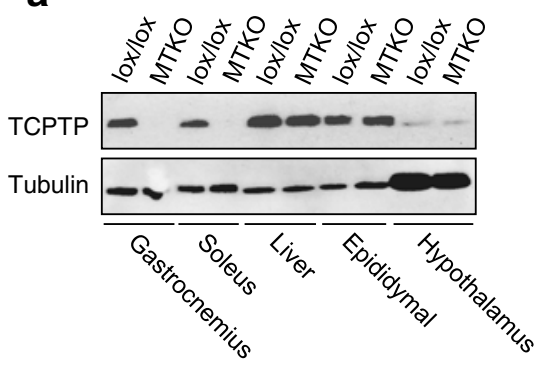

b

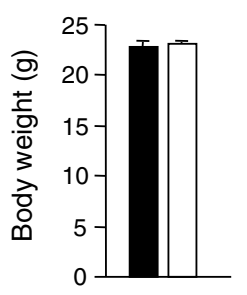

C

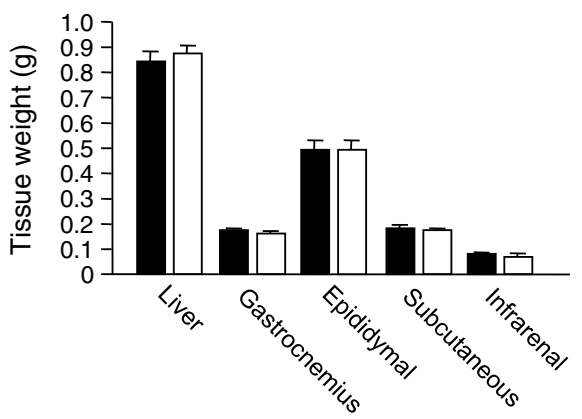

\section{d}
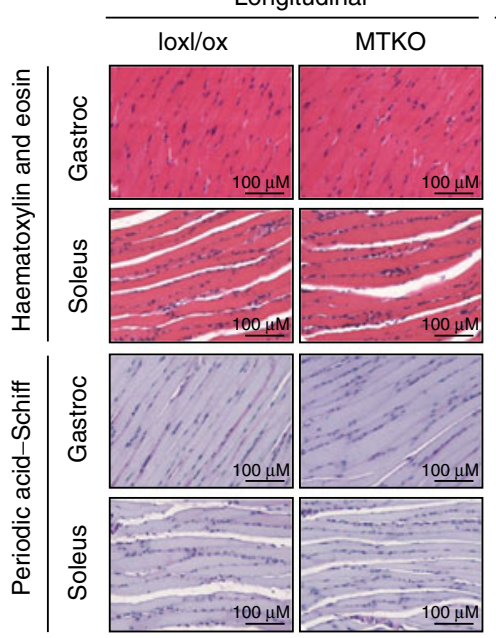

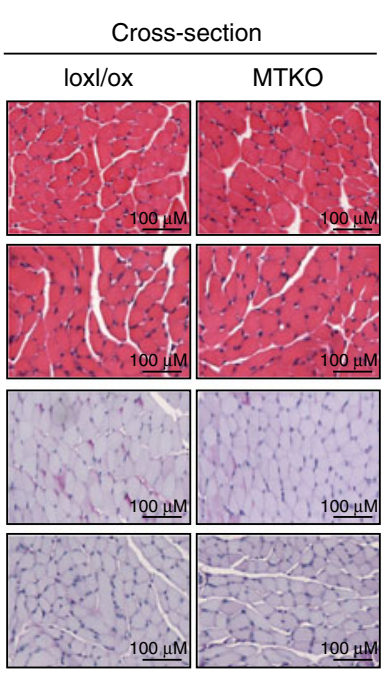

e
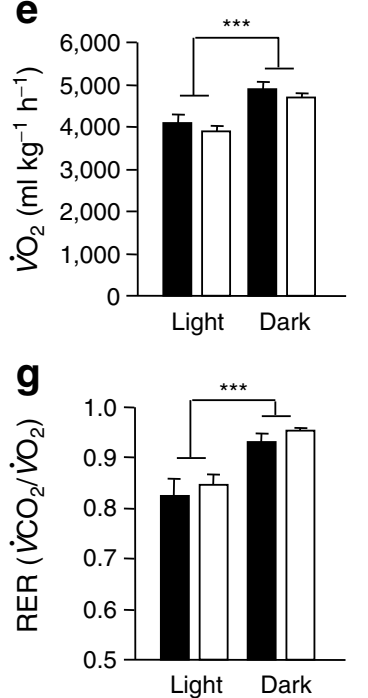
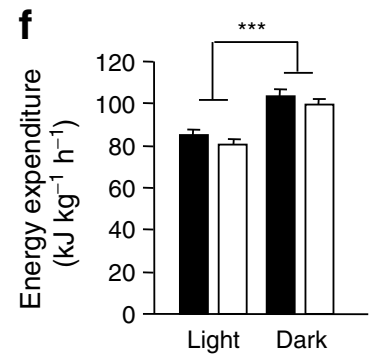

h

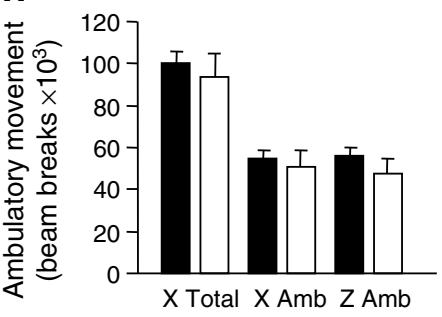

Fig. 1 Generation and characterisation of muscle-specific TCPTPdeficient mice. a TCPTP levels in Ptpn $2^{\text {lox/lox }}$ control (lox/lox) and Mck-Cre;Ptpn $2^{\text {lox/lox }}$ muscle-specific TCPTP-deficient (MTKO) mice as determined by immunoblotting. b Body weight in 8-week-old and (c) tissue weight in 8- to 10 -week-old male lox/lox (black bars, $n=7$ ) and MTKO (white bars, $n=7$ ) mice. d Gastrocnemius (Gastroc) and soleus muscle from lox/lox and MTKO mice were isolated and

be evident when mice are fed a high-fat diet to render them insulin-resistant. For example, mice with a muscle-specific deficiency in phosphatase and tensin homologue (PTEN), a PTP superfamily member with lipid phosphatase activity that attenuates PI3K signalling, do not exhibit any overt differences in insulin sensitivity unless challenged with a high-fat diet [40]. Therefore, we next examined whether muscle-specific TCPTP deficiency could enhance insulin sensitivity and prevent both weight gain induced by a high-fat diet and the development of insulin resistance. Mck-Cre; $P t p n 2^{\text {lox/lox }}$ and Ptpn $2^{\text {lox/lox }}$ male mice (8-10-week-old) were fed a normal chow $(4.6 \%$ fat) or a high-fat ( $23 \%$ fat) diet for 12 weeks. No differences in weight gain or adiposity were evident in chow-fed or HFF Mck-Cre;Ptpn $2^{\text {lox/lox }}$ versus Ptpn $2^{\text {lox/lox }}$ mice (Fig. 4a-f). Similarly no differences in oxygen consumption, energy expenditure or fuel utilisation (respiratory exchange ratios) were noted in chow-fed (data processed for histological analysis (staining as indicated). Scale bars, $100 \mu \mathrm{m}$. e Oxygen consumption, (f) energy expenditure and (g) respiratory exchange ratios (RER) in the light and dark cycles in 8- to 10-week-old lox/lox and MTKO mice were determined by indirect calorimetry. $\mathbf{h}$ Ambulatory activity ( $x$ and $z$ axes) was also determined. b, c, e-h Results are mean $\pm \mathrm{SE}$; $(\mathbf{e}-\mathbf{g}) * * * p<0.001$ for light cycle versus dark cycle

not shown) or HFF Mck-Cre;Ptpn $2^{\text {lox/lox }}$ versus Ptpn $2^{\text {lox/lox }}$ mice (Fig. 4g-i). There was a trend for lower ambulatory activity in HFF Mck-Cre;Ptpn $2^{\text {lox/ox }}$ mice (Fig. 4j), but this was not statistically significant $(p=0.11)$.

To determine whether muscle-specific TCPTP deficiency could attenuate the development of insulin resistance induced by a high-fat diet, we performed ITTs and GTTs. No significant differences in insulin sensitivity or glucose tolerance were observed in 8-week-old Mck-Cre;Ptpn $2^{\text {lox/lox }}$ versus Ptpn $2^{\text {lox/lox }}$ male mice that had been fed a chow or a high-fat diet for 12 weeks (Fig. 5a-h). In addition, fed and fasted blood glucose levels were similar between Mck-Cre; Ptpn $2^{\text {lox/lox }}$ and Ptpn $2^{\text {lox } / o x}$ mice on either diet (Fig. 5i-j). Fed and fasted plasma insulin levels were also unaltered in HFF Mck-Cre;Ptpn $2^{\text {lox/lox }}$ versus Ptpn2 ${ }^{\text {lox } / l o x}$ mice (Fig. 5k). Finally, insulin-induced muscle Akt Ser-473 phosphorylation was not different in HFF Mck-Cre;Ptpn $2^{l o x / l o x}$ versus 

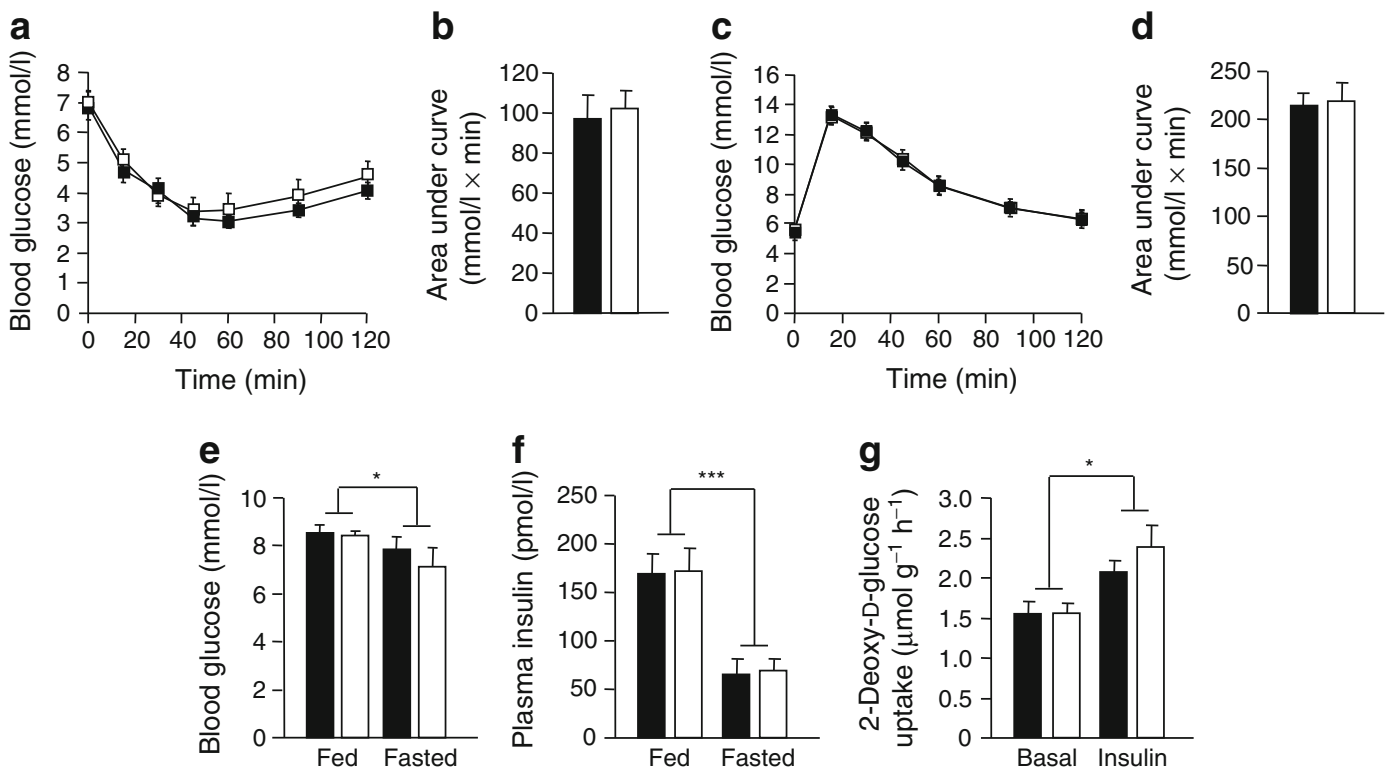

Fig. 2 Glucose homeostasis in muscle-specific TCPTP-deficient (MTKO) mice. a Male lox/lox (black symbols/bars) and MTKO (white symbols/bars) mice (8-10 weeks old) were fasted and ITTs (insulin $0.5 \mathrm{mU} / \mathrm{g}$ body weight) performed, with (b) determination of AUC. c GTTs (glucose $1 \mathrm{mg} / \mathrm{g}$ body weight) were performed and (d)

Ptpn $2^{\text {lox/lox }}$ mice (Fig. 51, m). Taken together, these results indicate that TCPTP deficiency in muscle has no overt effect on insulin signalling and glucose homeostasis, and does not prevent the development of insulin resistance associated with high-fat feeding.

TCPTP and PTP1B are not redundant in insulin signalling The unaltered insulin signalling in TCPTP-deficient muscle might be due to a functional redundancy with PTP1B. To explore this possibility, we investigated whether the combined inhibition of TCPTP and PTP1B in muscle cells might result in synergistic or additive effects on insulin signalling (Fig. 6). To inhibit TCPTP and/or PTP1B, we
AUC determined in mice as above (a, b). e Fed and fasted blood glucose and (f) plasma insulin levels in mice as above (a, b). g 2-Deoxy-D- $\left[{ }^{3} \mathrm{H}\right]$ glucose uptake assays of control and insulinstimulated soleus explants from lox/lox and MTKO mice. Results are mean \pm SE; $n=7$ all groups; ${ }^{*} p<0.05$ and ${ }^{* * *} p<0.001$

used the highly selective inhibitors, compound 8 ( $K_{\mathrm{i}}$ value TCPTP $4.3 \mathrm{nmol} / 1$ [38]) and compound II ( $K_{\mathrm{i}}$ value PTP1B $26 \mathrm{nmol} / 1$ [41]), respectively. Consistent with previous studies implicating PTP1B in the regulation of insulin signalling in muscle $[11-13,15]$, we found that PTP1B, but not TCPTP inhibition enhanced insulin-induced PI3K/Akt signalling in rat L6 myoblasts (Fig. 6a, b). TCPTP inhibition with compound 8 resulted in increased phosphorylation of STAT3 (Fig. 6c), a bona fide TCPTP substrate [8, 32]. However, no additive effects on insulin signalling (as assessed by Akt Ser-473 phosphorylation) were evident in L6 cells when PTP1B and TCPTP were both inhibited (Fig. 6b). These results thus indicate that



Fig. 3 Insulin signalling in muscle-specific TCPTP-deficient (MTKO) mice and muscle cells. a Male lox/lox and MTKO mice aged 8 to 10 weeks were fasted for $4 \mathrm{~h}$ and injected with saline or insulin $(1 \mathrm{mU} / \mathrm{g}, 10 \mathrm{~min}$, i.p.), after which gastrocnemius muscle was extracted and processed for immunoblot analysis with antibodies to the phosphorylated (Y1162/Y1163) and activated insulin receptor $\beta$ b

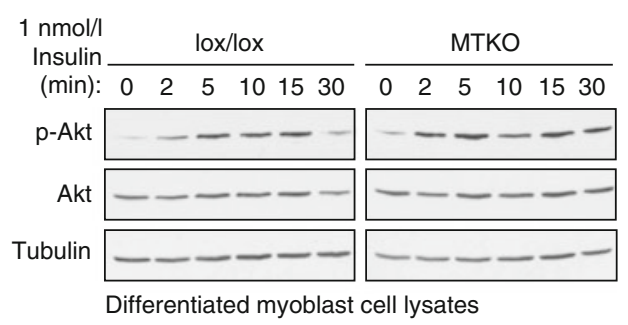

subunit (p-IR) and Ser-473 phosphorylated Akt (p-Akt), and then with antibodies to total insulin receptor (IR) and Akt. b Myoblasts from lox/lox and MTKO mice were differentiated, serum-starved and stimulated with $1 \mathrm{nmol} / \mathrm{l}$ insulin for the indicated times and processed for immunoblot analysis as indicated. Results are representative of three independent experiments 

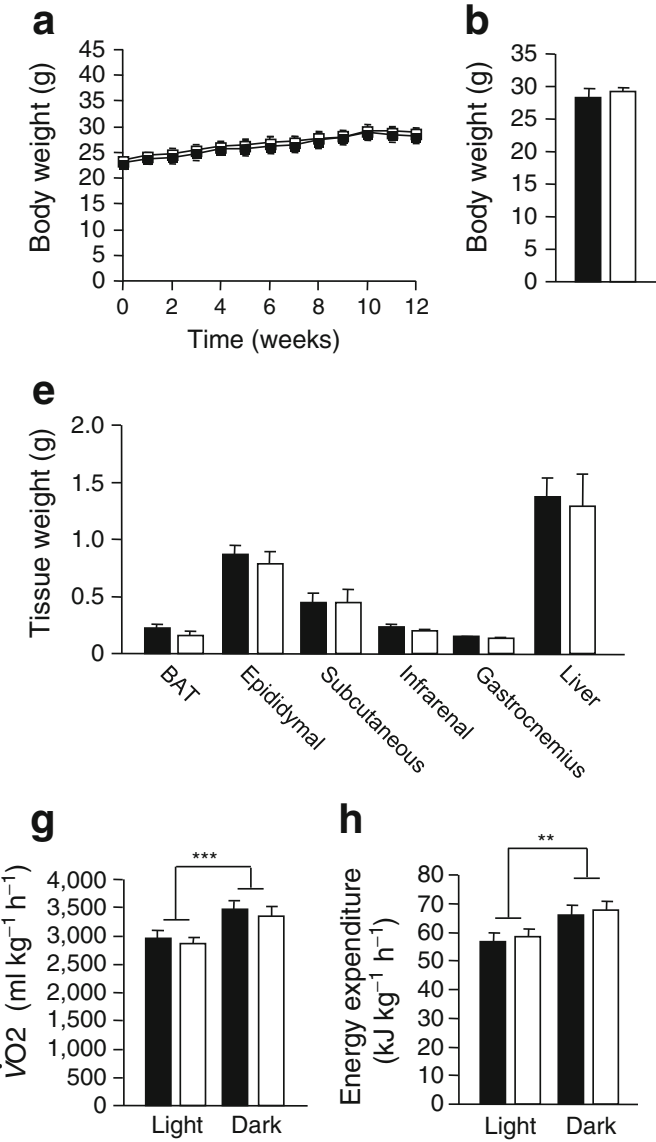

Fig. 4 Body weight, adiposity and energy homeostasis in musclespecific TCPTP-deficient (MTKO) mice. a Male lox/lox (black symbols/ bars) and MTKO (white symbols/bars) mice aged 8 to 10 weeks were fed a normal chow diet ( $n=6$ per genotype) for 12 weeks and body weight measured weekly, with (b) final weight given for 12 weeks. c Mice as above (a) were fed a high-fat diet ( $n=7$ per genotype) and body weight measured, with final measurement (d) at 12 weeks. e Tissue

TCPTP does not regulate insulin-induced PI3K/Akt signalling in muscle cells, even when PTP1B is inhibited.

\section{Discussion}

Although we have shown previously that the insulin receptor can serve as a substrate for TCPTP $[8,10,29$, $30,32]$, in this study we report that TCPTP does not regulate insulin receptor signalling in muscle. In contrast to PTP1B deficiency, which enhances insulin signalling and glucose uptake in muscle, and protects mice from insulin resistance $[11,12,15]$, we found that TCPTP deficiency in muscle had no overt effect on glucose homeostasis.

The catalytic domains of PTP1B and TCPTP share a high degree of primary ( $72 \%$ identity, $86 \%$ similarity) and tertiary structural similarity, and have similar active sites. In
C

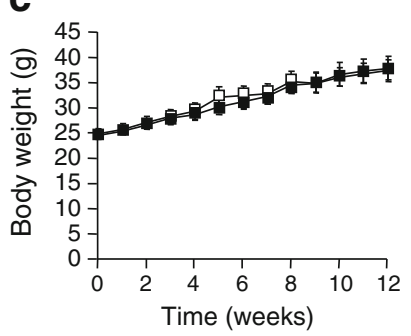

d

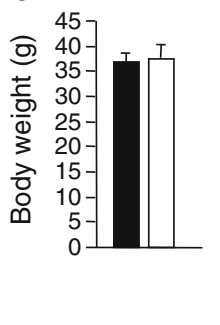

f

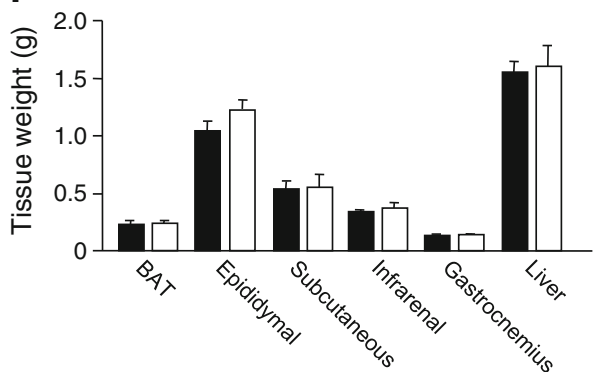

i
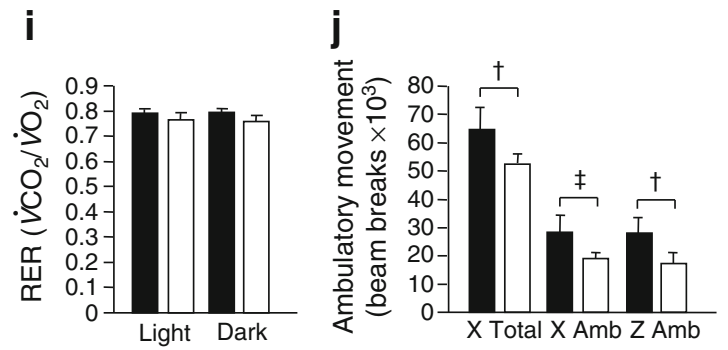

weight in chow-fed (12 weeks, $n=6$ per genotype) and (f) HFF (12 weeks, $n=7$ per genotype) lox/lox and MTKO mice. g Oxygen consumption, (h) energy expenditure, (i) respiratory exchange ratios (RER) and (j) ambulatory activity were determined in HFF lox/lox and MTKO mice $(n=6$ per genotype). Results $(\mathbf{a}-\mathbf{j})$ are mean \pm SE; ${ }^{* *} p<0.01,{ }^{* * *} p<0.001,{ }^{\dagger} p<0.11,{ }^{*} p<0.14$

particular, both have a second phosphotyrosine-binding pocket that allows for the selective recognition of tandem phosphorylated substrates [42, 43] such as the insulin receptor PTK [10, 29, 42, 44], janus kinase (JAK) PTKs [44] and the met proto-oncogene tyrosine kinase (MET) receptor PTK [45]. However, despite their similarity, TCPTP and PTP1B exhibit a high degree of substrate selectivity. For example, PTP1B can dephosphorylate JAK2, but not JAK1/3, whereas TCPTP dephosphorylates JAK1/3, but not JAK2 [44, 46, 47]. These differences in PTP1B versus TCPTP substrate selectivity are associated with inherent differences in PTP catalytic domain substrate specificity, as well as with differences in tissue distribution and subcellular localisation. PTP1B is ubiquitous, whereas TCPTP is most abundant in the haematopoietic compartment [8]. Moreover, PTP1B is targeted to the endoplasmic reticulum by a hydrophobic C-terminus, whereas TCPTP is produced as two variants, one targeted to the endoplasmic 
a
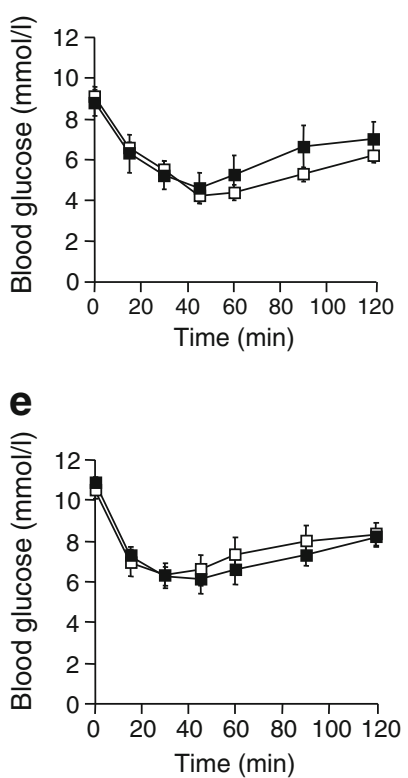

i

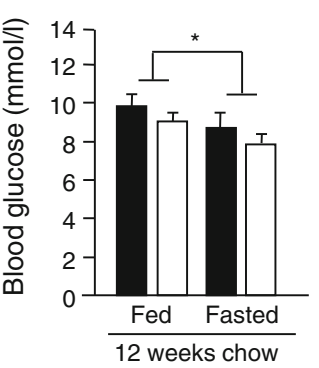

I

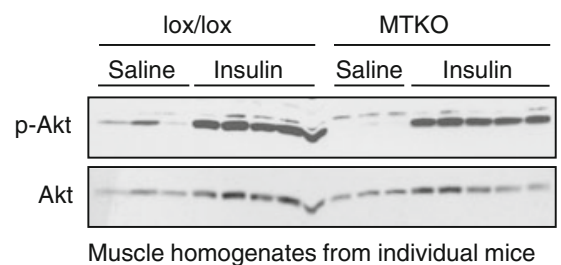

b

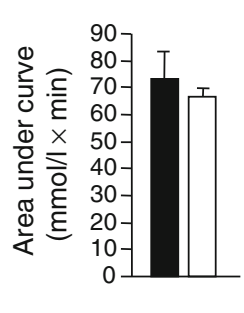

f

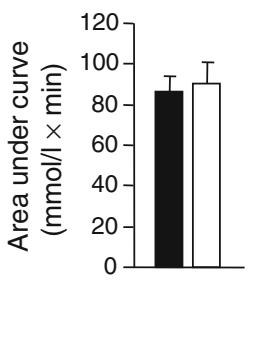

C
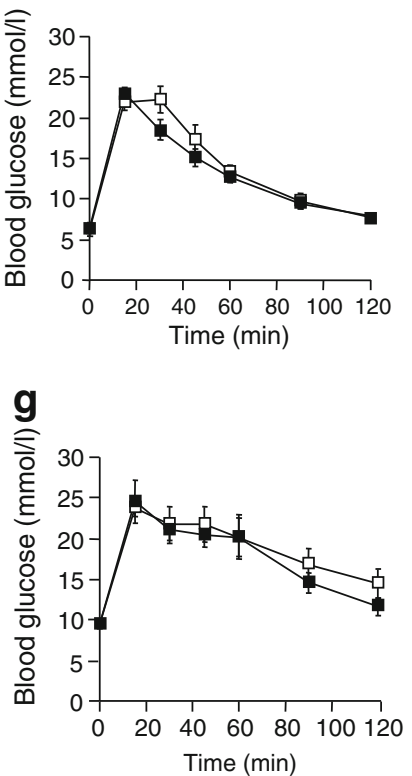

k

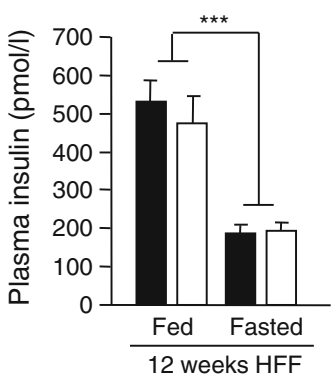

d

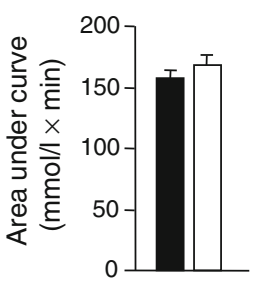

h

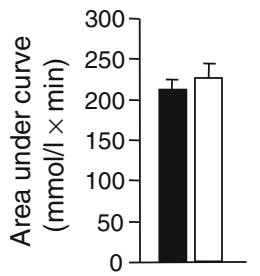

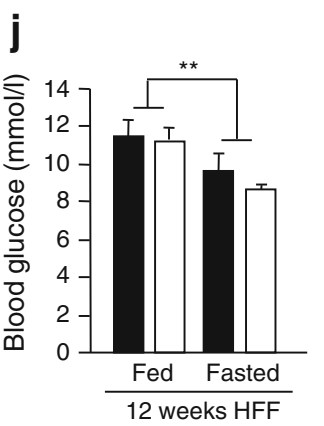

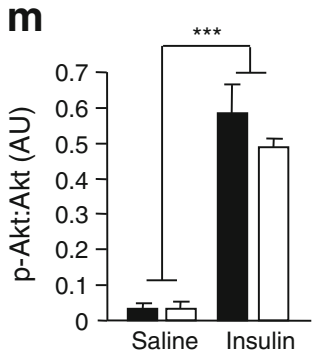

Fig. 5 Glucose homeostasis in muscle-specific TCPTP-deficient (MTKO) mice fed a high-fat diet. Male lox/lox (black symbols/bars) and MTKO (white symbols/bars) mice aged 8 to 10 weeks were fed a normal chow or a high-fat diet for 12 weeks. a Mice on chow diet were fasted and ITTs (insulin $0.75 \mathrm{mU} / \mathrm{g}$ body weight) were performed, with (b) determination of AUC. c GTTs (glucose $2 \mathrm{mg} / \mathrm{g}$ body weight) were performed on mice as above (a), and (d) AUC determined. e ITT results and (f) AUC for HFF mice at 12 weeks; (g) GTT results and (h) AUC for HFF mice at 12 weeks. a-h $n=7$ per genotype. Fed and fasted blood glucose levels in (i) chow-fed $(n=6$

reticulum and the other to the nucleus [8]; both TCPTP variants are produced in muscle [32].

In keeping with the differences in substrate specificity exhibited by these two phosphatases, we have shown per genotype) and (j) HFF ( $n=7$ per genotype) lox/lox and MTKO male mice after 12 weeks of respective diets. $\mathbf{k}$ Fed and fasted plasma insulin levels in HFF mice. I HFF mice (saline, $n=3$ per genotype; insulin, $n=5$ per genotype) were fasted for $4 \mathrm{~h}$ and injected with saline or insulin $(0.75 \mathrm{mU} / \mathrm{g}$; i.p.), after which gastrocnemius muscle was extracted and processed for immunoblot analysis with antibodies to phosphorylated (p)-Akt, Akt and tubulin. m p-Akt was quantified by densitometry and normalised to total Akt, expressed in arbitrary units (AU). Results $(\mathbf{a}-\mathbf{m})$ are mean $\pm \mathrm{SE} ;{ }^{*} p<0.05, * * p<0.01,{ }^{* * *} p<0.001$

previously that PTP1B and TCPTP can function cooperatively in the same cell to regulate the intensity and duration of insulin receptor activation and signalling [10, 29, 30]. Similarly, PTP1B and TCPTP work in concert to regulate 


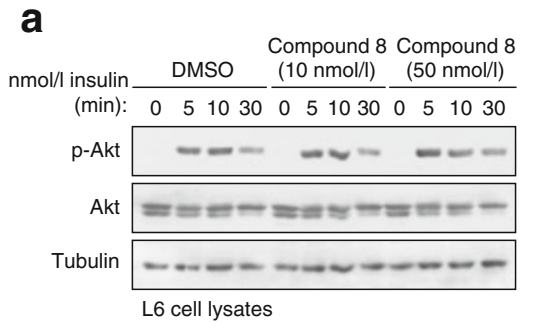

b

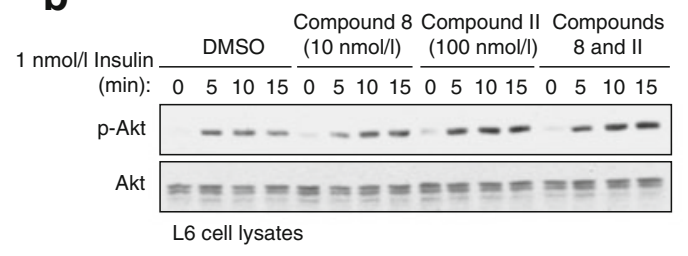

C



Fig. 6 PTP1B but not TCPTP inhibition in muscle cells enhances insulin signalling. L6 myoblasts were serum-starved and pre-incubated for $1 \mathrm{~h}$ with (a) 10 and $50 \mathrm{nmol} / 1$ compound 8 (TCPTP inhibitor), and (b) $10 \mathrm{nmol} / 1$ compound 8, $100 \mathrm{nmol} / 1$ compound II (PTP1B inhibitor), or both, followed by stimulation with $1 \mathrm{nmol} / 1$ insulin for the indicated times and processing for immunoblot analysis with antibodies to phosphorylated (p)-Akt, Akt and tubulin. c L6 cells were pretreated with vehicle control or $10 \mathrm{nmol} / 1$ compound 8 , and stimulated with $10 \mathrm{ng} / \mathrm{ml} \mathrm{IL}-6$ for the indicated time, followed by processing for immunoblot analysis with antibodies to p-STAT3 and STAT3. Results are representative of three independent experiments
MET receptor phosphorylation [45] and also regulate STAT6 phosphorylation in the cytoplasm and nucleus, respectively [48]. Thus, PTP1B and TCPTP can function in a coordinated manner for the temporal and spatial control of cellular signalling. Both TCPTP [32] and PTP1B [33] appear to be required for insulin receptor regulation in the liver. In other studies, we have shown that chow-fed liverspecific TCPTP-deficient mice exhibit enhanced glucose homeostasis (T. Tiganis, unpublished observation). So why might TCPTP be redundant in insulin receptor activation and signalling in muscle? One possibility is that relative TCPTP abundance might be decreased and/or PTP1B abundance increased in muscle, but this does not appear to be the case [32, 33]; indeed PTP1B levels are lower in muscle than in liver [33]. Moreover, we have shown here that TCPTP deficiency does not enhance insulin signalling, even when PTP1B is inhibited, arguing against functional redundancy due to the presence of PTP1B.

The evidence that PTP1B regulates insulin receptor activation and signalling in muscle is compelling [11, 12, 15]. In particular, insulin sensitivity is increased in chowfed muscle-specific Ptpn1-knockout mice, and muscle insulin receptor and IRS-1 phosphorylation are elevated in the fasted state [15]. Consistent with this, our studies indicate that PTP1B inhibition in muscle cells can enhance insulin signalling. However, recent studies have indicated that PTP1B, but not TCPTP may affect the endosomal sorting of activated receptor PTKs through the dephosphorylation of proteins such as signal transducing adaptor molecule (SH3 domain and ITAM motif) 2 (STAM2) [49]. Therefore one possibility is that the effects of PTP1B on insulin signalling may be indirect and attributable to altered endosomal sorting of the insulin receptor. In this case, PTPs other than PTP1B and TCPTP may be responsible for the direct dephosphorylation of the insulin receptor in response to insulin. One candidate may be $\mathrm{PTP} \varepsilon$, which translocates to the insulin receptor in response to insulin [28]. Another candidate is SHP-1, the global deletion of which results in a dramatic improvement in insulin sensitivity and glucose tolerance, and a significant enhancement of insulin-induced insulin receptor and IRS phosphorylation in muscle [50].

The inhibition of PTP1B to enhance insulin responsiveness and alleviate insulin resistance remains a rational therapeutic approach for the treatment of type 2 diabetes [21]. However, PTP1B inhibitors in preclinical development also inhibit TCPTP to varying degrees [21]. From our previous work, the question has arisen of whether the inadvertent inhibition of TCPTP by PTP1B inhibitors in muscle might contribute to the enhancement of insulin sensitivity; our current studies suggest that this is unlikely. Additional studies are required to determine whether TCPTP has a role in other insulin-responsive tissues such as fat, where PTP1B may be redundant in regulating the insulin response [12, 13].

Acknowledgements We thank C. Yang and T. Tiganis for technical support. This work was supported by the National Health and Medical Research Council (NHMRC) of Australia (to T. Tiganis and M.J. Watt) and the National Institutes of Health (to Z.-Y. Zhang, RO1 CA126937; and B.G. Neel, R37 CA49152), and by funds from the Ontario Ministry of Health and Long Term Care and the Princess Margaret Hospital Foundation (to B.G. Neel). T. Tiganis and M.J. Watt are NHMRC Research Fellows; B.G. Neel is a Canada Research Chair (Tier I).

Duality of interest Z.-Y. Zhang is a co-founder of Aarden Pharmaceuticals and Chairman of its scientific advisory board. The authors declare that there is no other duality of interest associated with this manuscript.

Contribution statement All authors contributed to the conception and design, or analysis and interpretation of data, and to the drafting or revising of the article critically for important intellectual content. All authors provided final approval of the version to be published. 


\section{References}

1. Saltiel AR, Kahn CR (2001) Insulin signalling and the regulation of glucose and lipid metabolism. Nature 414:799-806

2. Biddinger SB, Kahn CR (2006) From mice to men: insights into the insulin resistance syndromes. Annu Rev Physiol 68:123-158

3. Bevan P (2001) Insulin signalling. J Cell Sci 114:1429-1430

4. White MF (1998) The IRS-signalling system: a network of docking proteins that mediate insulin action. Mol Cell Biochem 182:3-11

5. Ng Y, Ramm G, Lopez JA, James DE (2008) Rapid activation of Akt2 is sufficient to stimulate GLUT4 translocation in 3T3-L1 adipocytes. Cell Metab 7:348-356

6. Bai L, Wang Y, Fan J et al (2007) Dissecting multiple steps of GLUT4 trafficking and identifying the sites of insulin action. Cell Metab 5:47-57

7. Cho H, Mu J, Kim JK et al (2001) Insulin resistance and a diabetes mellitus-like syndrome in mice lacking the protein kinase Akt2 (PKB beta). Science 292:1728-1731

8. Tiganis T, Bennett AM (2007) Protein tyrosine phosphatase function: the substrate perspective. Biochem J 402:1-15

9. Cheng A, Dube N, Gu F, Tremblay ML (2002) Coordinated action of protein tyrosine phosphatases in insulin signal transduction. Eur J Biochem 269:1050-1059

10. Galic S, Hauser C, Kahn BB et al (2005) Coordinated regulation of insulin signaling by the protein tyrosine phosphatases PTP1B and TCPTP. Mol Cell Biol 25:819-829

11. Elchebly M, Payette P, Michaliszyn E et al (1999) Increased insulin sensitivity and obesity resistance in mice lacking the protein tyrosine phosphatase-1B gene. Science 283:1544-1548

12. Klaman LD, Boss O, Peroni OD et al (2000) Increased energy expenditure, decreased adiposity, and tissue-specific insulin sensitivity in protein-tyrosine phosphatase 1B-deficient mice. Mol Cell Biol 20:5479-5489

13. Bence KK, Delibegovic M, Xue B et al (2006) Neuronal PTP1B regulates body weight, adiposity and leptin action. Nat Med 12:917-924

14. Haj FG, Zabolotny JM, Kim YB, Kahn BB, Neel BG (2005) Liver specific protein-tyrosine phosphatase $1 \mathrm{~B}$ (PTP1B) re-expression alters glucose homeostasis of PTP1B-/-mice. J Biol Chem 280:15038-15046

15. Delibegovic M, Bence KK, Mody $N$ et al (2007) Improved glucose homeostasis in mice with muscle-specific deletion of protein-tyrosine phosphatase 1B. Mol Cell Biol 27:7727-7734

16. Zabolotny JM, Haj FG, Kim YB et al (2004) Transgenic overexpression of protein-tyrosine phosphatase $1 \mathrm{~B}$ in muscle causes insulin resistance, but overexpression with leukocyte antigen-related phosphatase does not additively impair insulin action. J Biol Chem 279:24844-24851

17. Ahmad F, Azevedo JL, Cortright R, Dohm GL, Goldstein BJ (1997) Alterations in skeletal muscle protein-tyrosine phosphatase activity and expression in insulin-resistant human obesity and diabetes. J Clin Invest 100:449-458

18. Ahmad F, Goldstein BJ (1995) Alterations in specific proteintyrosine phosphatases accompany insulin resistance of streptozotocin diabetes. Am J Physiol 268:E932-E940

19. Goldstein BJ (2002) Protein-tyrosine phosphatases: emerging targets for therapeutic intervention in type 2 diabetes and related states of insulin resistance. J Clin Endocrinol Metab 87:2474-2480

20. Zabolotny JM, Kim YB, Welsh LA, Kershaw EE, Neel BG, Kahn BB (2008) Protein-tyrosine phosphatase 1B expression is induced by inflammation in vivo. J Biol Chem 283:14230-14241

21. Zhang S, Zhang ZY (2007) PTP1B as a drug target: recent developments in PTP1B inhibitor discovery. Drug Discov Today $12: 373-381$
22. Swarbrick MM, Havel PJ, Levin AA et al (2009) Inhibition of protein tyrosine phosphatase-1B with antisense oligonucleotides improves insulin sensitivity and increases adiponectin concentrations in monkeys. Endocrinology 150:1670-1679

23. Hashimoto N, Feener EP, Zhang WR, Goldstein BJ (1992) Insulin receptor protein-tyrosine phosphatases. Leukocyte common antigen-related phosphatase rapidly deactivates the insulin receptor kinase by preferential dephosphorylation of the receptor regulatory domain. J Biol Chem 267:13811-13814

24. Ahmad F, Considine RV, Goldstein BJ (1995) Increased abundance of the receptor-type protein-tyrosine phosphatase LAR accounts for the elevated insulin receptor dephosphorylating activity in adipose tissue of obese human subjects. J Clin Invest 95:2806-2812

25. Kulas DT, Zhang WR, Goldstein BJ, Furlanetto RW, Mooney RA (1995) Insulin receptor signaling is augmented by antisense inhibition of the protein tyrosine phosphatase LAR. J Biol Chem 270:2435-2438

26. Zhang WR, Li PM, Oswald MA, Goldstein BJ (1996) Modulation of insulin signal transduction by eutopic overexpression of the receptor-type protein-tyrosine phosphatase LAR. Mol Endocrinol 10:575-584

27. Zabolotny JM, Kim YB, Peroni OD et al (2001) Overexpression of the LAR (leukocyte antigen-related) protein-tyrosine phosphatase in muscle causes insulin resistance. Proc Natl Acad Sci U S A 98:5187-5192

28. Aga-Mizrachi S, Brutman-Barazani T, Jacob AI, Bak A, Elson A, Sampson SR (2008) Cytosolic protein tyrosine phosphataseepsilon is a negative regulator of insulin signaling in skeletal muscle. Endocrinology 149:605-614

29. Galic S, Klingler-Hoffmann M, Fodero-Tavoletti MT et al (2003) Regulation of insulin receptor signaling by the protein tyrosine phosphatase TCPTP. Mol Cell Biol 23:2096-2108

30. Meng TC, Buckley DA, Galic S, Tiganis T, Tonks NK (2004) Regulation of insulin signaling through reversible oxidation of the protein-tyrosine phosphatases TC45 and PTP1B. J Biol Chem 279:37716-37725

31. Tiganis $\mathrm{T}$ (2011) Reactive oxygen species and insulin resistance: the good, the bad and the ugly. Trends Pharmacol Sci 32:82-89

32. Fukushima A, Loh K, Galic S et al (2010) T cell protein tyrosine phosphatase attenuates STAT3 and insulin signaling in the liver to regulate gluconeogenesis. Diabetes 59:1906-1914

33. Delibegovic M, Zimmer D, Kauffman C et al (2009) Liverspecific deletion of protein-tyrosine phosphatase 1B (PTP1B) improves metabolic syndrome and attenuates diet-induced ER stress. Diabetes 58:590-599

34. Loh K, Fukushima A, Zhang X et al (2011) Elevated hypothalamic TCPTP in obesity contributes to cellular leptin resistance. Cell Metab 14:684-699

35. Loh K, Deng H, Fukushima A et al (2009) Reactive oxygen species enhance insulin sensitivity. Cell Metab 10:260-272

36. Hansen PA, Wang W, Marshall BA, Holloszy JO, Mueckler M (1998) Dissociation of GLUT4 translocation and insulinstimulated glucose transport in transgenic mice overexpressing GLUT1 in skeletal muscle. J Biol Chem 273:18173-18179

37. Xie L, Lee SY, Andersen JN et al (2003) Cellular effects of small molecule PTP1B inhibitors on insulin signaling. Biochemistry 42:12792-12804

38. Zhang S, Chen L, Luo Y, Gunawan A, Lawrence DS, Zhang ZY (2009) Acquisition of a potent and selective TC-PTP inhibitor via a stepwise fluorophore-tagged combinatorial synthesis and screening strategy. J Am Chem Soc 131:13072-13079

39. Tiganis T, Bennett AM, Ravichandran KS, Tonks NK (1998) Epidermal growth factor receptor and the adaptor protein $\mathrm{p} 52^{\text {Shc }}$ are specific substrates of T cell protein tyrosine phosphatase. Mol Cell Biol 18:1622-1634 
40. Wijesekara N, Konrad D, Eweida M et al (2005) Muscle-specific Pten deletion protects against insulin resistance and diabetes. Mol Cell Biol 25:1135-1145

41. Xie L, Zhang YL, Zhang ZY (2002) Design and characterization of an improved protein tyrosine phosphatase substrate-trapping mutant. Biochemistry 41:4032-4039

42. Salmeen A, Andersen JN, Myers MP, Tonks NK, Barford D (2000) Molecular basis for recognition and dephosphorylation of the activation segment of the insulin receptor by protein tyrosine phosphatase 1B. Mol Cell 6:1401-1412

43. Iversen LF, Moller KB, Pedersen AK et al (2002) Structure determination of $\mathrm{T}$ cell protein tyrosine phosphatase. J Biol Chem 277:19982-19990

44. Simoncic PD, Lee-Loy A, Barber DL, Tremblay ML, McGlade CJ (2002) The $\mathrm{T}$ cell protein tyrosine phosphatase is a negative regulator of janus family kinases 1 and 3. Curr Biol 12:446-453

45. Sangwan V, Paliouras GN, Abella JV et al (2008) Regulation of the Met receptor-tyrosine kinase by the protein-tyrosine phosphatase 1B and T cell phosphatase. J Biol Chem 283:3437434383

46. Myers MP, Andersen JN, Cheng A et al (2001) TYK2 and JAK2 are substrates of protein-tyrosine phosphatase 1B. J Biol Chem 276:47771-47774

47. Zabolotny JM, Bence-Hanulec KK, Stricker-Krongrad A et al (2002) PTP1B regulates leptin signal transduction in vivo. Dev Cell 2:489-495

48. Lu X, Malumbres R, Shields B et al (2008) PTP1B is a negative regulator of interleukin 4-induced STAT6 signaling. Blood 112:4098-4108

49. Stuible M, Abella JV, Feldhammer M et al (2010) PTP1B targets the endosomal sorting machinery: dephosphorylation of regulatory sites on the endosomal sorting complex required for transport component STAM2. J Biol Chem 285:23899-23907

50. Dubois MJ, Bergeron S, Kim HJ et al (2006) The SHP-1 protein tyrosine phosphatase negatively modulates glucose homeostasis. Nat Med 12:549-556 